\title{
A Hybrid Cutting Approach for Hysteroscopy Simulation
}

\author{
M. Harders ${ }^{1}$, D. Steinemann ${ }^{2}$, M. Gross ${ }^{2}$, and G. Székely ${ }^{1}$ \\ Swiss Federal Institute of Technology \\ ${ }^{1}$ Computer Vision Lab \\ ${ }^{2}$ Computer Graphics Lab \\ ETH Zentrum, CH-8092 Zürich, Switzerland \\ \{mharders, szekely\}@vision.ee.ethz.ch, \{deniss, grossm\}@inf.ethz.ch
}

\begin{abstract}
An integral element of every surgical simulator is the ability to interactively cut tissue. A number of approaches have been suggested in the past, the most important being mesh subdivision by introducing new elements and mesh adaptation by adjusting existing topology. In this paper we combine these two methods and optimize them for our training system of hysteroscopic interventions. The basic methodology is introduced in $2 \mathrm{D}$, a first extension to $3 \mathrm{D}$ is presented and finally the integration into the simulator described.
\end{abstract}

\section{Introduction}

Therapeutic hysteroscopy has become a common technique in gynecological practice 4]. Nevertheless, a number of potentially dangerous complications exist - the most common being uterine wall perforation, intra-uterine bleeding, and mismanagement of distension fluid. In [19] the rate of complications for therapeutic interventions is reported as 17\%. According to 14, 97\% of hysteroscopic interventions are performed with resectoscopes, with up to $9 \%$ of them leading to perforations. The most critical situation is wall perforation with resectoscope electrodes during cutting procedures, since lesions of intra-abdominal organs are likely. In these cases hysteroscopy usually has to be stopped and an emergency abdominal intervention performed. Therefore, specialized training is necessary to reduce the rate of complications. Virtual Reality based surgical simulation [11] is one option to provide a corresponding learning environment. In contrast to existing systems and products [10 15 13], our work aims at achieving the highest possible realism. With this system, we intend to identify the necessary level of fidelity for achieving a specific training effect, by stepwise reduction of realism. As described above, a key element of training is the tissue ablation process. We currently focus on the resection of intra-uterine neoplasms. Myomectomy with loop electrodes has to be carried out by stepwise shaving. The process is depicted in Figure 3. Including these procedures in our simulator system necessitates updates of the underlying tissue model.

Different approaches for handling cutting have been proposed, which can be summarized into three major categories: straight-forward element deletion 
[2/7/5], mesh subdivision [12 16/8/31] and topology adaptation [20]17]. While the first method is not appropriate for a realistic simulator, the latter two have produced reasonable results. However, both of them still have significant limitations (e.g. increase of element count, reduced element sizes or degenerate elements), which will be discussed in more detail in the next section. In the following, we propose a hybrid approach, combining subdivision with mesh adaptation strategies. These are completed by a subsequent mesh optimization step. Our method is tailored for our hysteroscopic training system.

\section{Previous Work}

Cutting approaches are usually tailored to the applied deformation and visualization mesh representations. With only few exceptions, these are triangular or tetrahedral meshes, respectively. Straight-forward deletion of mesh entities has been applied in [2] and [5]. The idea is to remove elements, which are contacted by a cutting tool. Unfortunately, this leads to visual artifacts, since the cutting path can not be accurately approximated. Moreover, the principle of mass conservation is violated.

More appealing visual representations of incisions were made possible with mesh subdivision methods. These usually have in common the classification of a cut according to different rotational invariant intersection states. Predefined subdivisions of mesh elements will then be performed. In the context of medical applications, this was first introduced in [12] for cutting of tetrahedral meshes with predefined planes. This approach has been refined in [16], where partial incision of mesh elements as well as progressive cutting is taken into account. Finally, [1 discusses the use of a state machine to keep track of different incisions in tetrahedral meshes. All described approaches have in common the often considerable increase of element count, which is reported to range from five up to 17 new elements per incised tetrahedron. Moreover, introduction of new mesh elements often necessitates extensive model recalculations, for instance when using implicit FEM. Another negative factor is the reduction in element sizes. Deformation stability problems were reported [16], which required a significant reduction of the time step, thus rendering real-time simulation intractable.

Some of these problems could be ameliorated with topology adaptation approaches as suggested in [20] and [17. The central idea is to approximate a cutting path with existing edges or surfaces. This enables mesh incisions without large increase of element count and occurrence of small elements. Unfortunately, problems arise due to degenerated elements, which can appear, if unconditional node displacement is carried out in the mesh. Also, the quality of incision approximation is limited by the initial mesh resolution.

\section{Hybrid Cutting Approach in 2D}

The key idea of our method is the combination of mesh adaptation by adjusting existing topology, and mesh subdivision by introducing new elements. This is 
complemented by subsequent local mesh optimization procedures to ensure a consistent overall mesh quality. Finally, similar deformation behavior is guaranteed by adjusting mechanical parameters. The basic concepts are first introduced in $2 \mathrm{D}$, however, the strength of our approach becomes especially evident in the $3 \mathrm{D}$ case.

Generally, element count \#E, number of nodes \#N and element size should be kept constant. Therefore, mesh adaptation should be applied whenever possible. To this end, edges are divided into regions, which define, whether displacement or subdivision is applied. Three general cases can occur as depicted in Figure 5. Depending on the intersection, one or two additional elements are created. In extreme circumstances, this still leads to an increase in element count. The situation, however, is rectified in the mesh optimization step described below. With the combined approach, small sized elements as well as degeneracies are avoided. Moreover, the process is easily applicable in progressive cutting, since only local edge intersection information is needed.

The method is further refined by extending the view beyond the current triangle. If two type 2 cases follow each other, element creation can be avoided with an edge flip. However, if the second displacement would involve a neighbor of the edge to be flipped, this can not be done. This case requires the addition of two new elements. Furthermore, a few other cases can appear, which need special treatment. Similar to the previous example, it can happen, that after a type 3 case, the displacement of a neighbor would require additional elements. This can be solved by displacing the node on the cut itself. The selected cases discussed are shown in Figure 4. Special treatment is also necessary for nodes on the object border. Displacement can only be performed within the border to preserve object shape. Moreover, this is only possible, if no prominent features, for instance sharp corners, are represented by the nodes. This is the case, if the angle between normals of neighboring border elements is small.

Succeeding several adaptation/subdivision steps, a mesh optimization process is performed. Although generally applicable, this step is optimized for a straight incision through several triangles. This is justified by the nature of the cutting process in myomectomy (see section 4). The optimization includes edge collapse and equal node distribution on the cut, as well as local mesh

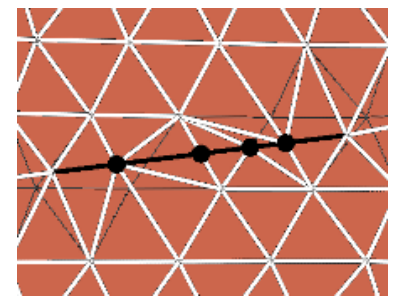

(a) Before optimization after initial cut.

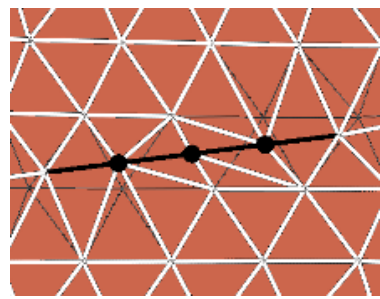

(b) Edge collapse and node distribution on cut.

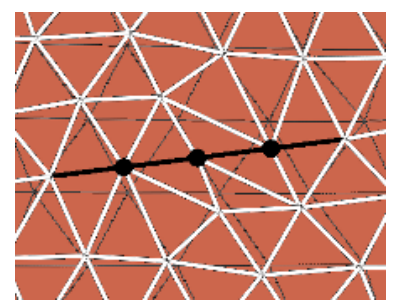

(c) Mesh after homogenization step.

Fig. 1. Mesh optimization process (cut line/nodes depicted in black) 
regularization. The latter is carried out by local computation of a combined mass-spring/particle system in the cut's vicinity. In this local system, nodes, which can be reached from cut nodes via one or two edge steps, are movable, while the remainder, including the cut nodes, is fixed. Mean local edge length is used to set mass-spring constants and particle system force profiles. Moreover, constant masses and high damping values are applied to avoid unnecessary oscillations. Positions $\boldsymbol{x}_{\tilde{\boldsymbol{i}}}$ of the nodes proximal to the cut are adjusted by integrating the equation of motion.

$$
m_{\tilde{i}} \ddot{\boldsymbol{x}}_{\tilde{i}}+c_{\tilde{i}} \dot{\boldsymbol{x}}_{\tilde{i}}+\left(\alpha \sum_{j} \boldsymbol{g}_{\tilde{i} j}+\beta \boldsymbol{f}_{\tilde{i}}^{i n t}\right)=0, \quad \tilde{i} \in \tilde{\mathcal{N}}
$$

where $\tilde{\mathcal{N}}$ are indices of the proximal nodes. $\boldsymbol{g}_{\tilde{i} j}$ are spring forces, and $\boldsymbol{f}_{\tilde{i}}^{i n t}$ are forces resulting from the potential energy function of the particle system. The homogenization will be carried out, until node movement $x_{\tilde{i}}^{t}-x_{\tilde{i}}^{t-1}$ drops below $2 \%$ of mean edge length. Generally, about $4 \tilde{n}$ steps have to be carried out, with $\tilde{n}$ being the number of elements intersected. All three optimization steps are displayed in Figure 1 .

Table1 shows results obtained with our proposed algorithm. The test meshes were created with the DistMesh tool described in [18. The following statistical values were determined: number of nodes and elements, minimum, mean and standard deviation of element areas $\left(A_{\min }, A_{\mu}, A_{\sigma}\right)$ and edge lengths $\left(E_{m i n}, E_{\mu}\right.$, $E_{\sigma}$ ), as well as mesh optimization time $t$. Moreover, a commonly used quality measure $q \in\left[0, \frac{1}{2}\right]\left(q_{\min }, q_{\mu}, q_{\sigma}\right)$ based on ratio between largest inscribed and smallest circumscribed circle, is also computed (triangles with $q>0.25$ are considered to be well shaped). All values were determined for the initial mesh (Initial), as well as after remeshing with standard subdivision (Stndrd) and our hybrid approach (Hybrid). With our proposed method element and node count were kept almost constant. Also, the values of $A_{\min }, E_{\min }$ and $q_{\text {min }}$, which have the strongest impact on deformation stability, remained favorable. Computation time increased in larger meshes, which is due to large incisions performed across

Table 1. Statistical values of test cases with two example meshes

\begin{tabular}{|c|c|c|c|c|c|c|c|c|c|c|c|c|c|}
\hline Test & & $\# \mathbf{N}$ & $\# \mathbf{E}$ & $A_{\min }$ & $\mathbf{A}_{\mu}$ & $\mathbf{A}_{\sigma}$ & $\mathbf{E}_{\min }$ & $\mathbf{E}_{\mu}$ & $\mathbf{E}_{\sigma}$ & $q_{\min }$ & $\mathbf{q}_{\mu}$ & $\mathbf{q}_{\sigma}$ & $\mathbf{t}[m s]$ \\
\hline & Initial & 88 & 59 & 88.93 & 113.64 & 11.06 & 12.63 & 16.26 & 1.81 & 0.393 & 0.473 & 0.028 & \\
\hline \multirow[t]{2}{*}{$1 a$} & Stndrd & 107 & 70 & 0.23 & 93.46 & 38.27 & 0.66 & 14.64 & 4.07 & 0.012 & 0.433 & 0.108 & \multirow[b]{2}{*}{10} \\
\hline & Hybrid & 88 & 59 & 72.45 & 113.64 & 18.31 & 10.30 & \begin{tabular}{|l|}
16.31 \\
\end{tabular} & \begin{tabular}{|l|}
2.25 \\
\end{tabular} & 0.361 & 0.465 & 0.032 & \\
\hline \multirow[t]{2}{*}{$1 \mathrm{~b}$} & Stndrd & 108 & 70 & 0.38 & \begin{tabular}{|r|}
92.59 \\
\end{tabular} & 37.72 & 0.87 & 14.62 & 3.97 & 0.036 & 0.434 & 0.101 & \multirow[b]{2}{*}{20} \\
\hline & Hybrid & 93 & 62 & 71.96 & 107.98 & 15.79 & 9.94 & 15.93 & 2.29 & 0.284 & 0.460 & 0.036 & \\
\hline & Initial & 519 & 298 & 4.10 & 5.09 & 0.28 & 2.75 & 3.45 & 0.27 & 0.398 & 0.487 & 0.015 & \\
\hline \multirow[t]{2}{*}{$2 \mathbf{a}$} & Stndrd & 587 & 335 & 0.03 & 4.50 & 1.40 & 0.05 & 3.23 & 0.68 & 0.011 & 0.461 & 0.086 & \multirow[b]{2}{*}{190} \\
\hline & Hybrid & 521 & 299 & 2.19 & 5.07 & 0.43 & 2.19 & 3.45 & 0.32 & 0.332 & 0.483 & 0.021 & \\
\hline \multirow[t]{2}{*}{$2 \mathrm{~b}$} & Stndrd & 603 & 343 & 0.03 & 4.39 & 1.50 & 0.09 & 3.18 & 0.72 & 0.012 & 0.454 & 0.094 & \multirow[b]{2}{*}{250} \\
\hline & Hybrid & 517 & 297 & 3.51 & 5.11 & 0.52 & 2.03 & 3.48 & 0.39 & 0.270 & 0.478 & 0.028 & \\
\hline
\end{tabular}


the complete mesh without any intermediate calculations. This can be alleviated by progressively carrying out regularization while the cut is being made.

Finally, we have to update the tissue model, since the underlying mesh has been changed. This is necessary to ensure a consistent deformation behavior. For the currently applied mass-spring system, node masses and spring constants need to be adjusted. The new mass distribution can be obtained via preservation of object mass moments $p_{j k}$ up to second order as suggested in [6]. The moments are computed for the initial configuration.

$$
p_{j k}=\sum_{i=1}^{n} x_{i}^{j} y_{i}^{k} m_{i} \quad j+k \leq 2
$$

where $\left(x_{i}, y_{i}\right)$ and $m_{i}$ are coordinates and masses of node $i$. This gives us the moment vector $\boldsymbol{p}=\left(p_{00}, p_{01}, \ldots, p_{20}\right)^{T}$ for the initial mesh. After the remeshing process, the new mass coefficients $\boldsymbol{m}^{*}$ for the adjusted node coordinates can the be obtained via the precomputed moments. To this end, we have to solve the underdetermined system $\boldsymbol{A} \boldsymbol{m}^{*}=\boldsymbol{p}$, where $\boldsymbol{A}$ is the Vandermonde matrix of the new coordinates. This can be done, by introducing $\boldsymbol{B}=\boldsymbol{A} \boldsymbol{A}^{\boldsymbol{T}}$. We first solve $\boldsymbol{B} \tilde{\boldsymbol{m}}=\boldsymbol{p}$, and in a second step, we obtain the new mass distribution according to $\boldsymbol{m}^{*}=\boldsymbol{A}^{\boldsymbol{T}} \tilde{\boldsymbol{m}}$.

For setting of new spring constants $k^{*}$, a method was suggested in [9].

$$
k_{s}^{*}=\frac{E \sum_{E} \operatorname{area}\left(T_{E}\right)}{|s|^{2}}
$$

where the sum is over elements $T_{E}$ incident on edge $s$ and $E$ is Young's modulus. This was suggested for isotropic, homogeneous, linear elastic material.

\section{Extension of Hybrid Cutting to 3D}

The main elements of our hybrid approach have been implemented for incisions into tetrahedral meshes. In the current stage, subsequent mesh optimization has not been integrated. However, the mass-spring/particle regularization generalizes

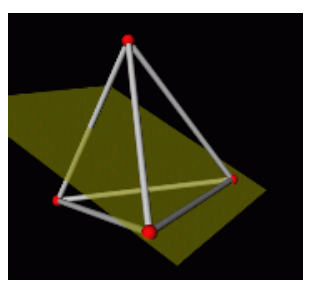

(a) Cut plane through one tetrahedron

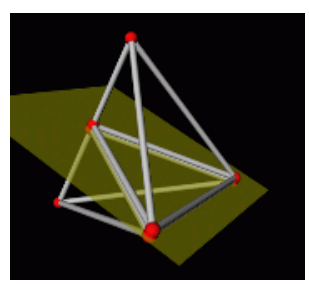

(b) After approach

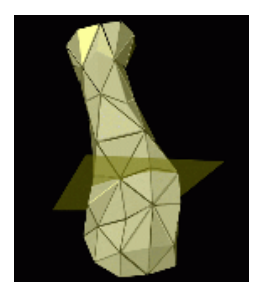

(c) Example mesh for a polyp

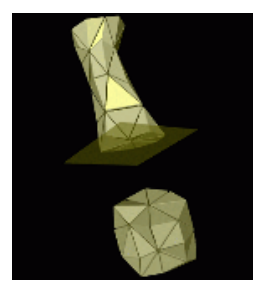

(d) Excised tissue sample

Fig. 2. Examples of incisions into 3D meshes 


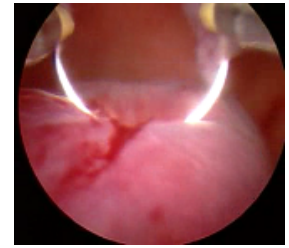

(a) Deformation with inactive electrode

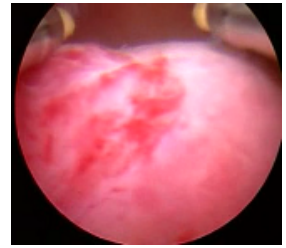

(b) Start of cut

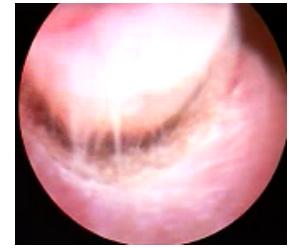

(c) Directly after end (d) of cut

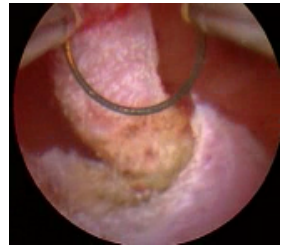

tissue moved against fundus

Fig. 3. Steps of shaving process with loop electrode during myomectomy

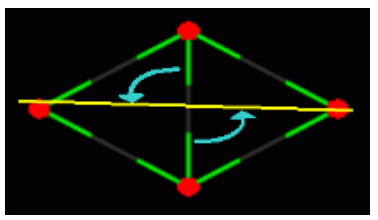

(a) Edge exchange possible in this configuration

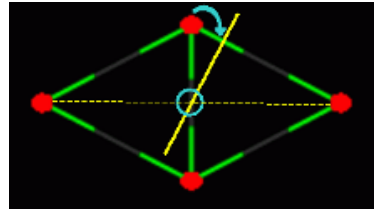

elements

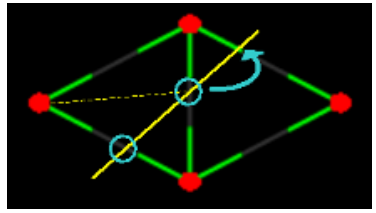

(c) Cut node displaced necessary

Fig. 4. Treatment of special cases

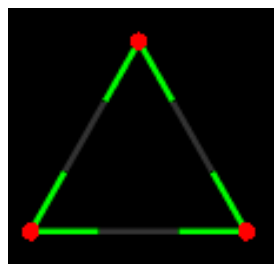

(a) Edge regions

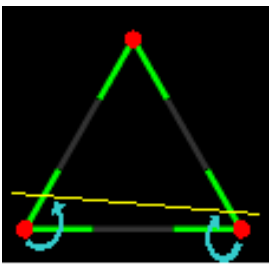

(b) Case 1: Two displacements

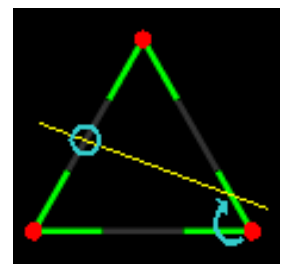

(c) Case 2: Subdivision \& displace

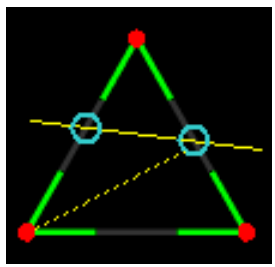

(d) Case 3: Two subdivisions

Fig. 5. Cases for hybrid approach (cut line depicted in yellow)

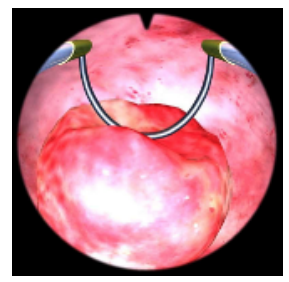

(a) Deformation with inactive electrode

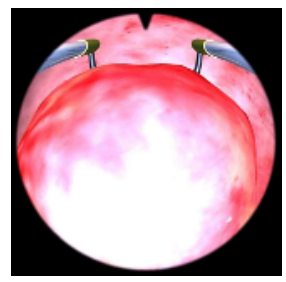

(b) Start of cut

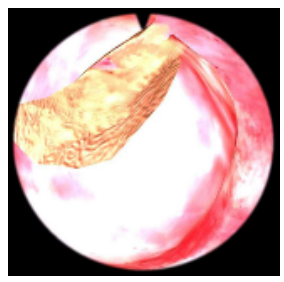

(c) Directly after end (d) Excised tisof cut

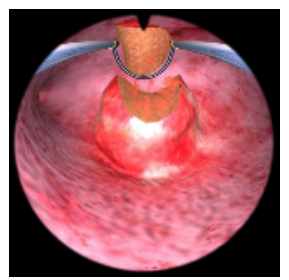

sue moved against fundus

Fig. 6. Steps of myoma shaving process in Virtual Reality 
Table 2. Statistical values of test cases with a tetrahedral mesh

\begin{tabular}{||c|r|r|r|r|r|r|r|r|r|r|r||}
\hline \hline Test & $\# \mathbf{N}$ & $\# \mathbf{E}$ & $\mathbf{V}_{\min }$ & $\mathbf{V}_{\mu}$ & $\mathbf{V}_{\sigma}$ & $\mathbf{E}_{\mathbf{m i n}}$ & $\mathbf{E}_{\mu}$ & $\mathbf{E}_{\sigma}$ & $\mathbf{q}_{\mathbf{m i n}}^{*}$ & $\mathbf{q}_{\mu}^{*}$ & $\mathbf{q}_{\sigma}^{*}$ \\
\hline \hline Initial & 69 & 136 & 3.54 & 37.07 & 15.80 & 3.14 & 7.19 & 1.99 & 0.017 & 0.21 & 0.06 \\
\hline Standard & 319 & 620 & $8.8 x 10^{-6}$ & 8.13 & 14.5 & 0.03 & 3.69 & 2.78 & $3.2 x 10^{-3}$ & 0.09 & 0.09 \\
\hline Hybrid A & 76 & 136 & 3.54 & 37.12 & 15.82 & 3.14 & 7.17 & 1.99 & 0.018 & 0.22 & 0.06 \\
\hline Hybrid B & 88 & 145 & 2.96 & 34.77 & 16.29 & 2.82 & 7.06 & 1.96 & 0.017 & 0.21 & 0.06 \\
\hline \hline
\end{tabular}

well to 3D and its addition is straight-forward. Again, subdivision is carried out, if a cut plane is close to the middle of an edge. If the plane is close to a node, the incision is approximated by separating existing mesh nodes. This gives us four rotationally invariant cases, where zero to three nodes of a tetrahedra are separated. Examples of this process can be seen in Figure 2.

As in 2D, this approach allows for smooth cuts without considerably increasing node and element count, while mesh quality is kept in an acceptable range. Table 2 shows statistical values for example cuts in 3D. It contains the number of nodes and elements, minimum, mean and standard deviation of element volumes $\left(V_{\min }, V_{\mu}, V_{\sigma}\right)$ and edge lengths. $q^{*} \in\left[0, \frac{1}{3}\right]$ is again a quality measure, this time denoting the ratio between radii of tetrahedra inspheres and circumspheres. All values were determined for the initial mesh, the mesh after remeshing with standard subdivision (Standard), as well as two different cuts using our hybrid approach $($ HybridA/B). Our approach prevents a considerable increase of element and node counts. The values of $V_{\min }, E_{\min }$ and $q_{\min }$, which have the strongest impact on deformation stability, remain again in acceptable ranges.

Since our application area is tissue ablation in hysteroscopy, we can make some assumptions about the cutting process. In the standard approach, the loop electrode is placed behind the pathology and then advanced towards the camera along a straight path (as depicted in Figure 3). This allows us to simplify the definition of the cutting surface by extruding the shape of the loop along the tool vector. Entry and exit position of the loop are registered in our collision detection routine. As soon as the tool leaves the tissue, our hybrid approach is carried out. Steps of this process, integrated into a simulator framework for hysteroscopy, are shown in Figure 6.

\section{Conclusion and Future Work}

We have presented a hybrid cutting approach combining mesh subdivision and topology adaptation. The approach was optimized for our specific application domain - simulation of tissue excision in hysteroscopic interventions. The method was introduced in $2 \mathrm{D}$, followed by an extension to 3D. Future work will focus on integration of the mesh regularization process also in 3D. Moreover, the generalization of the cutting approach to arbitrary cut paths will be further investigated. 


\section{Acknowledgment}

The authors would like to thank all developers of the hysteroscopy simulator project. This research has been supported by the NCCR Co-Me of the Swiss National Science Foundation.

\section{References}

1. D. Bielser et al. A state machine for real-time cutting of tetrahedral meshes. In Proc. of Pacific Graphics, pages 377-386, 2003.

2. M. Bro-Nielsen. Finite element modeling in medical VR. Journal of the IEEE, 86(3):490-503, 1998.

3. C. Bruyns and K. Montgomery. Generalized interactions using virtual tools within the spring framework: Cutting. In Proc. MMVR02, pages 23-26, 2002.

4. ACOG Tech. Bulletin. Hysteroscopy. Int J Gyn. Obstet., May 1994. 45(2): 175-180.

5. S. Cotin, H. Delingette, and N. Ayache. A hybrid elastic model allowing real-time cutting, deformations and force-feedback for surgery training and simulation. The Visual Computer, 16(8):437-452, 2000.

6. Oliver Deussen et al. Using simulated annealing to obtain good nodal approximations of deformable objects. In Comp. Anim. and Simul., pages 30-43, 1995.

7. S. Frisken-Gibson. Using linked volumes to model object collisions, deformation, cutting, carving, and joining. IEEE Trans on Vis. and Comp. Graphics, 5(4), 1999.

8. F. Ganovelli et al. A multiresolution model for soft objects supporting interactive cuts and lacerations. Computer Graphics Forum, 19(3), 2000.

9. A. Van Gelder. Approximate simulation of elastic membranes by triangulated spring meshes. Journal of Graphics Tools, 3(2):21-42, 1998.

10. J.S. Levy. Virtual reality hysteroscopy. J Am Assoc Gyn. Laparosc., 3 (4, Suppl.):25-26, 1996.

11. A. Liu et al. A survey of surgical simulation: Applications, technology, and education. Presence: Teleoperators \& Virtual Environments, 12(6):599-614, 2003.

12. A. Mazura and S. Seifert. Virtual cutting in medical data. In Proc. of Medicine Meets Virtual Reality, pages 420-429., 1997.

13. Immersion Medical. AccuTouch system. Company webpage (visited Mar 2005).

14. L. Mencaglia and E. Hamou. Manual of gynecological hysteroscopy - diagnosis and surgery. Endo-Press, Germany, 2001.

15. K. Montgomery et al. Surgical simulator for hysteroscopy: A case study of visualization in surgical training. In IEEE Visualization, 2001.

16. A. Mor and T. Kanade. Modifying soft tissue models: Progressive cutting with minimal new element creation. In MICCAI Proc., pages 598-607, 2000.

17. H.W. Nienhuys and A.F. van der Stappen. A surgery simulation supporting cuts and finite element deformation. In MICCAI'01 Proc., pages 153-160, 2001.

18. P.O. Persson and G. Strang. A simple mesh generator in matlab. SIAM Review, 46(2):329-345, 2004.

19. S.B. Pinion et al. Randomised trial of hysterectomy, endometrial laser ablation, and transcervical endometrial resection for dysfunctional uterine bleeding. British Medical Journal, 309:979-983, October 1994.

20. D. Serby, M. Harders, and G. Szekely. A new approach to cutting into finite element models. In MICCAI'01 Proc., pages 425-433, 2001. 